\title{
Intelligent and efficient development of wireless networks: A review of cognitive radio networks
}

\author{
ZHANG Ping*, LIU Yang, FENG ZhiYong, ZHANG QiXun, LI Qian \& XU Ding \\ Wireless Technology Innovation Institute (WTI), Key Laboratory of Universal Wireless Communications, Ministry of Education, Beijing \\ University of Posts and Telecommunications, Beijing 100876, China
}

Received November 10, 2011; accepted May 10, 2012

\begin{abstract}
Facing the challenges on how to improve spectrum efficiency and how to realize heterogeneous network convergence in future wireless networks, a cognitive radio network (CRN) is proposed as one of the solutions. This has become a major research topic in recent years and it is timely to give an overview of the development of CRN and to summarize key issues and technologies. The fundamental concepts of CRN, including the cognitive cycle model, the network architecture, and the cognitive ability and intelligent decision functions, are introduced in detail based on recent advances. Key issues for each topic, followed with recent research on theory and method, are then classified and the industrialization developments of CRN testbeds based on TD-LTE cellular system and standards are briefly presented. Finally, conclusions are reached on the perspectives and directions of future development.
\end{abstract}

cognitive radio networks, network architecture, cognitive ability, spectrum sensing, intelligent decision and dynamic spectrum allocation, end-to-end performance

Citation: Zhang P, Liu Y, Feng Z Y, et al. Intelligent and efficient development of wireless networks: A review of cognitive radio networks. Chin Sci Bull, 2012, 57: 3662-3676, doi: 10.1007/s11434-012-5334-5

Driven by the requirements of ubiquitous wireless access and personalized wireless applications [1], worldwide, numerous wireless technologies are invading our lives, such as evolutionary telecommunication technologies, wideband wireless access and short distance wireless technologies. Furthermore, the concept of the internet of things (IoT) has become widespread based on the development of wireless sensor networks.

With the explosive development of wireless technologies, several challenges must be addressed. The electromagnetic spectrum, whose use is licensed and managed by government, has been scarce for allocation to ubiquitous wireless applications. However, contrary to the physical scarcity of the available spectrum, the report published by the federal communication commission (FCC) shows that over $60 \%$ of the licensed spectrum below $6 \mathrm{GHz}$ remains underused [2,3]. The measurement results in Figure 1 taken in Beijing over a

\footnotetext{
*Corresponding author (email: pzhang@bupt.edu.cn)
}

one month period, also indicate low spectrum use consistent with the results released by the FCC. Therefore, improvement of spectrum efficiency is the first challenge to be examined. On the other hand, the end-to-end performance of wireless networks is considered to be the performance indicator for ubiquitous and personalized wireless applications [4], such as end-to-end throughput, end-to-end delay and routing scheme, which take into account all network elements in a data transmission flow across different layers for the global optimization from the network aspect. Because the property of heterogeneity causes low radio resource use and mutual interference among heterogeneous networks, the other big challenge is that heterogeneous network convergence is needed to improve the end-to-end performance of wireless networks.

Cognitive radio (CR), proposed by Mitola in 1999 [5,6], is considered a feasible solution for improving spectrum efficiency. Furthermore, a cognitive radio network (CRN), which is defined as a wireless network with the capabilities 


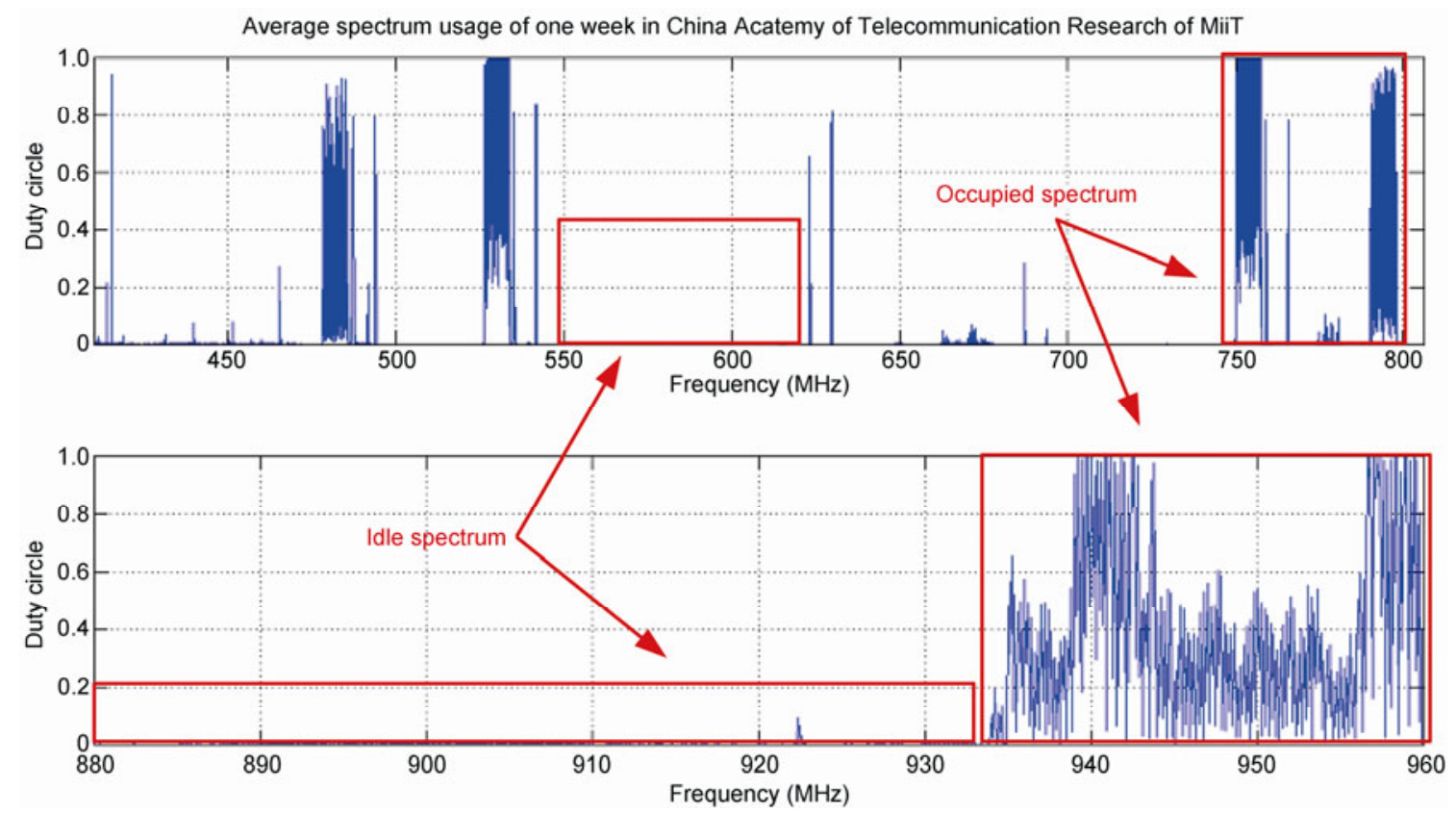

Figure 1 (Color online) Measurement of spectrum usage in Beijing.

of radio environment awareness, autonomous decision making, adaptive reconfigurating its infrastructure [7], and intelligent learning from experience of a continuously changing environment, is proposed to solve the challenges of efficient radio resource use and heterogeneous network convergence, with the aim of improving the end-to-end performance of wireless networks.

In recent years, CRN has received increasing attention. The current popular topics in CRN such as the framework of the cognitive function, spectrum sensing and dynamic spectrum access have been investigated in [8-10] respectively. To enable researchers to clearly understand the overall framework and development of $\mathrm{CRN}$, recent advances are introduced in this review.

\section{The fundamentals of CRN}

The fundamentals of CRN are introduced including the cognition cycle, network architecture and development of application scenarios.

\subsection{Cognition cycle for $\mathrm{CRN}$}

The research on CRN is mainly derived from the concept of a cognition cycle, in which the wireless network processes are mapped according to cognition theory by Mitola [5]. Besides, from Haykin's view, it focuses on four fundamental tasks: radio-scene analysis, channel identification, reconfiguration of transmit-power control and dynamic spectrum management [11]. Moreover, the cognition cycle proposed by Virginia Tech emphasizes the functions of reasoning and learning [12]. However, all of them focus only on the performance of the wireless transceiver, which is not enough to guarantee the end-to-end performance of the CRN from a network perspective. Therefore, the cognition cycle for $\mathrm{CRN}$ is proposed as shown in Figure 2, which includes four key aspects in CRN: end-to-end performance, cognitive ability, autonomous decision making and adaptive ability.

In the cognition cycle of CRN, first, there are many network elements for each end-to-end link in the transmission of data flow, in which each element has its requirements for personalized services. However, the overall network resource is limited by system bandwidth, available channel, transmit power, routing path, etc., so there are always tradeoffs between personalized user requirements, changeable wireless network environments, limited overall network resources and policies. This tradeoff for all elements in the end-to-end link can be modeled as the end-to-end performance, which is considered to be the performance indicator for the operation of elements involved in the end-to-end link.

CRN must have the ability to gather the necessary network information such as the available spectrum and operation parameters of the network, which is referred to as cognitive ability. Cognitive ability can enable a CRN to be aware of necessary information, such as the available spectrum, operation mode of the wireless network, transmitted waveform, network protocol, geographical information, type of services, user needs, and security policy. The CRN analyzes the information, and makes the decision to optimize the end-to-end performance of the wireless network, which can be characterized as an autonomous decision. Based on the optimized decision, the CRN can reconfigure 


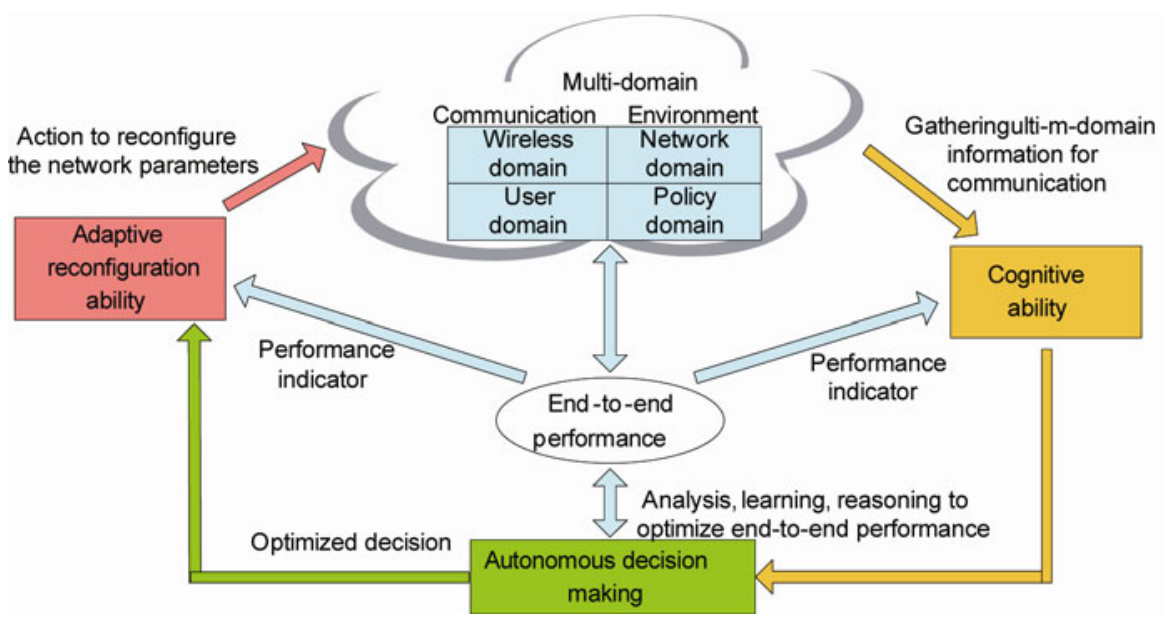

Figure 2 (Color online) Cognition cycle for a CRN.

its network parameters for operation, which is supported by the adaptive reconfiguration ability of the CRN. Therefore, the functions of the CRN for the cognition cycle are summarized as:

(i) Cognitive ability: Gather multi-domain information for communication such as wireless domain, network domain, user domain and policy domain.

(ii) Autonomous decision: Make autonomous decision to optimize the end-to-end performance of a wireless network. The network information and optimized decision can be learnt and reasoned as knowledge. The decision mainly relates to the reconfiguration operation for optimized radio resource management in a heterogeneous networks environment.

(iii) Adaptive reconfiguration ability: The network architecture of CRN should support the functions of the cognition cycle. It can adjust and reconfigure the network operation parameters, such as network working modes, network topologies, system protocols, and radio parameters, based on optimized decisions.

\subsection{Network architecture of CRN}

Radio architecture is a framework with which evolving families of components may be integrated into an evolving sequence of designs that synthesize specified functions within specified constraints (design rules) [13]. The network architecture of CRNs should have the adaptive ability that can support the functions of CRN, e.g. gathering network information, dynamic spectrum allocation, and spectrum hand off, etc. Thus, the components and communication protocols of CRN architecture should be designed according to the cognition cycle prototype and to enhance the end-to-end performance.

The network architecture of CRN refers to the control mode, network elements and protocol layers. The control mode can be classified as a centralized, distributed or hybrid mode. As in Figure 3 [14], the network components can be classified as primary components of primary base stations and users, cognitive components of cognitive base stations and users, and the spectrum broker which is responsible for spectrum tradeoff among different networks. Furthermore, protocol layers in CRNs are proposed, while the core functions of cognitive ability, autonomous decision making, and reconfiguration are integrated into a traditional OSI network protocol layers model.

The design of a "spectrum mobility function" requires reconfiguration decision making and network information exchange with other layers. Apparently, some decisions need to be based on joint information from more than one layer. Thus, the cross layer design should be considered in CRN.

In the past decade, many studies have focused on the network architecture of CRN. In [5], Mitola first developed an ideal architecture, in which user-awareness, selfawareness, and machine learning are considered to be key embedded functions. Reasoning components are incorporated in the architecture to make the plans and decisions more intelligent in wireless applications. In [11], the architecture proposed by Haykin emphasizes an awareness of the radio environments to operate in primary spectrum bands. The above two architectures are the most popular, and in addition there are studies on the network architecture of CRNs in the literature focusing on specific research areas [15-18].

In Figure 4, a network architecture of CRN is proposed, which comprises four functional building blocks, i.e., cognitive information management (CIM), database \& intelligent management (DB \& IM), network convergence management (NCM) and reconfiguration management (RM). CIM is responsible for gathering, processing and delivering cognitive information, which includes three sub-functions, i.e., cognitive information gathering (CIG), cognitive information processing (CIP) and cognitive information delivering (CID). DB \& IM is used to perform learning and save the results and long-term cognitive information in the 


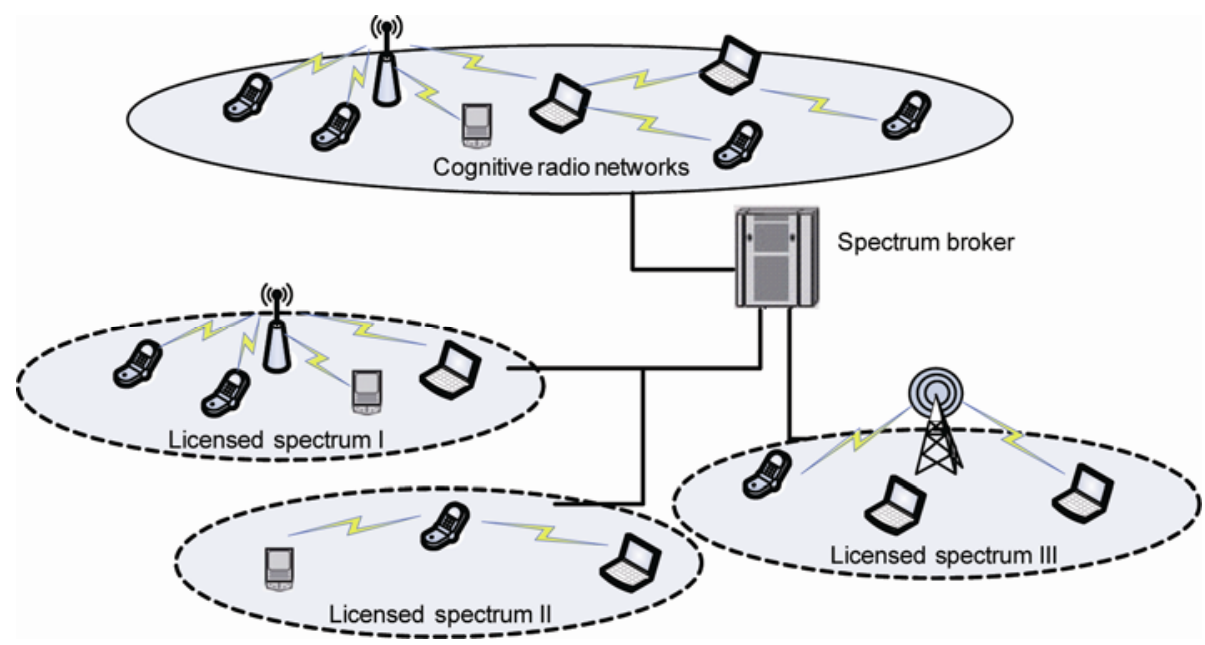

Figure 3 (Color online) CRN network architecture sketch map [14].

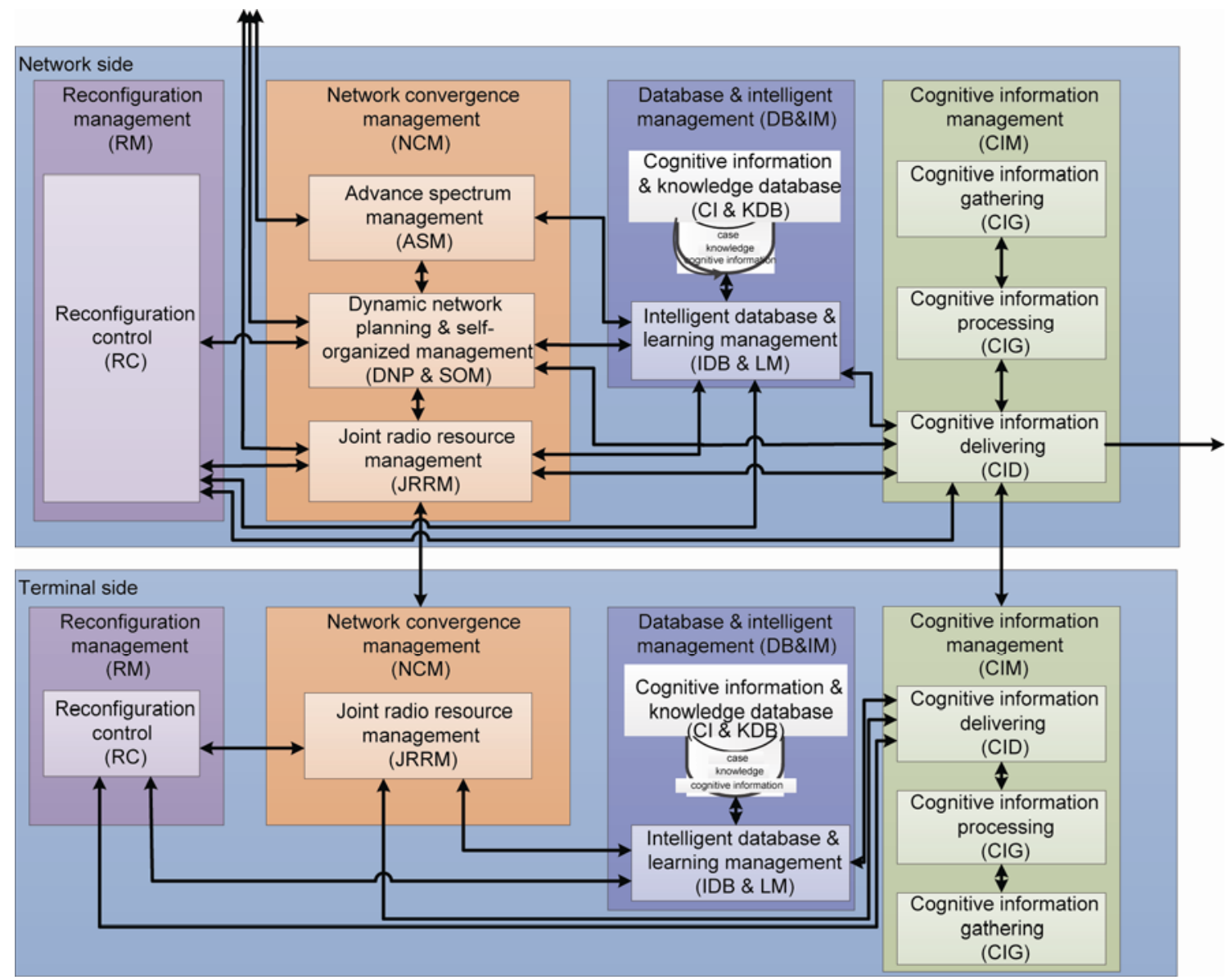

Figure 4 (Color online) Proposed network architecture of a CRN.

cognitive information \& knowledge database (CI \& KDB), where intelligent database \& learning management (IDB \& $\mathrm{LM}$ ) is used for learning process form experiences. NCM is mainly responsible for the coordination of devices to make optimized decisions to improve resource utilization in CRNs. It consists of three functional building blocks: ad- vance spectrum management (ASM), dynamic network planning and self-organized management (DNP \& SOM) and joint radio resource management (JRRM). ASM is mainly responsible for dynamic spectrum management among different heterogeneous networks, while DNP \& SOM is mainly responsible for network planning and 
self-organizing functions, such as the self-configuration, self-optimization, and self-healing functions. JRRM is mainly responsible for optimizing radio resource utilization among base stations and eNode B in heterogeneous networks environment. RM, which includes reconfiguration control (RC) function, is used to perform adaptive reconfigurations according to the optimized decisions made by NCM. Various interfaces between these functional building blocks are also defined to fulfill the demands of information exchanges, which are omitted for simplicity. It is noted that cognitive ability, autonomous decision, and adaptive reconfiguration ability have been integrated into this network architecture.

\subsection{Development of an application scenario}

A CRN can efficiently use network resources and optimize the end-to-end performance of a network so that it can be deployed in many application scenarios.

Dynamic spectrum access (DSA) is one of the most important application scenarios, as shown in Figure 5 [14]. The spectrum can be dynamically and flexibly shared between primary and secondary users in an opportunistic manner by utilizing the spectrum holes in time and frequency domains, so that the spectrum efficiency can be improved. Many researchers have focused on this topic [19-21]. Besides, DSA can also be applied in different heterogeneous cellular networks by dynamically sharing vacant spectrum in the intra-operator scenario. Moreover, the applications of military and public safety scenarios have special requirements for flexible spectrum access and interoperability between various wireless systems. A CRN can alleviate the spectrum congestion through efficient spectrum allocation and flexible spectrum access to adapt to the requirements and conditions of various wireless systems. Thus, a CRN can provide applications for cellular networks, military and public safety applications with adaptive, seamless, and secure wireless services for multiple service types, such as voice, data, and video.

Furthermore, many novel ideas based on CRNs have been proposed. For example, being applied in the emerging area of vehicular communication, in which carbon emissions in vehicular transport may be significantly reduced, and

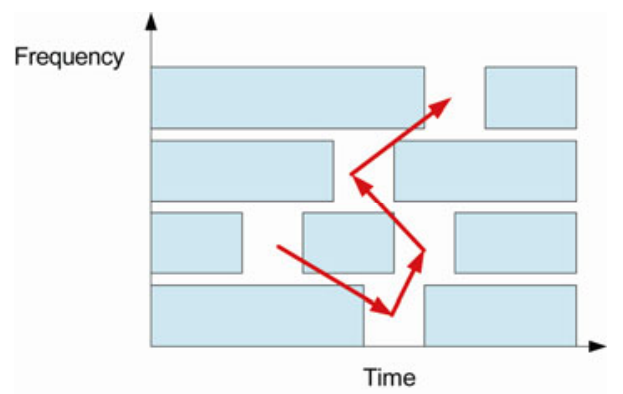

Figure 5 (Color online) Typical scenario for the use of a CRN [14]. vehicular transport may become safer through vehicles predicting collisions and taking appropriate corrective action [22]. Another important application is the commercial market for spectrum auction, such as the recent $700 \mathrm{MHz}$ spectrum auctions in the United States [23]. All of these applications drive the development of CRNs for commercial implementations. Besides, the CRN can easily change its configuration parameters by software to accommodate new applications. As long as no end-user hardware needs to be updated, product upgrades or configuration changes can be completed by simply downloading newly released software [23]. Therefore, the new wireless application can mainly be realized via software configuration and upgrade.

\section{Cognitive ability}

According to the analysis above, cognitive ability is the first step of a CRN in gathering the necessary network information such as available spectrum and network operation parameters. Cognitive ability can be mainly categorized as spectrum sensing, database and cognitive pilot channel (CPC) according to the different categories of information and collection methods. Spectrum sensing determines the available spectrum parameters including frequency, bandwidth, and idle period. The database and CPC can be used to collect or exchange the network information such as the radio access technology (RAT) mode, network pilot channel information, system bandwidth, carrier frequency, transmit power, and policies. The database stores and manages the information according to its properties, and the CPC transmits it via broadcast, multicast or on-demand mode according to the requirements of users.

\subsection{Spectrum sensing}

Spectrum sensing is defined as the task of finding spectrum holes by sensing the radio spectrum in the local neighborhood of the CRN in an unsupervised manner [24]. Spectrum holes, i.e. spectrum opportunities, are defined as "a band of frequencies that are not being used by the primary user of that band at a particular time in a particular geographic area" [25]. The spectrum holes can be modeled in the dimensions of frequency, time and space. Furthermore, there are other dimensions for spectrum holes, such as the code and angle [26]. Thus, the objective of spectrum sensing focuses mainly on the multi-dimensional properties of spectrum for exploring more spectrum holes.

To be specific, the task of spectrum sensing can be summarized as:

(i) Detection of spectrum holes: spectrum sensing detects the spectrum holes, and models them from the multi-dimensional properties based on the requirement of reliable communication for secondary users;

(ii) Interference analysis and signal classification: a sig- 
nal is detected and analyzed to classify it according to its characteristics. The interference can be distinguished from signals via characteristic analysis so that the CRN can reconfigure to avoid harmful interference to and from primary users.

To achieve these tasks above, numerous technologies are proposed to capture spectrum sensing which are reviewed below:

(i) Local detection. This focuses on the detection of the appearance of the primary user's signal based on signal processing methods. The sensing node (secondary base station or users) analyzes the received primary signal, and decides whether primary users are present or not. It has the challenge of limiting the transmit power of secondary devices to avoid interference to primary users. Thus, the FCC quantifies the measurement metric as the interference temperature for interference assessment [27], and an interference temperature threshold is proposed to denote the maximum amount of tolerable interference for a given frequency band at a particular location. The secondary users are required to transmit under the constraint that their transmission plus the existing noise and interference must not exceed the interference temperature threshold at a licensed receiver or primary user. The three main categories of local detection are energy detection, feature detection and matched filter detection.

(1) Energy detection: Energy detection is the most common method for spectrum sensing due to its low implementation complexity. It requires no prior knowledge of the primary user' signal, and does not need a special design for detecting spread spectrum signals [28]. Based on a comparison between the output of the energy detector and the given threshold for primary users, the challenges for energy detection are the selection of the appropriate detection threshold, the inability to differentiate interference from primary users and noise, and the poor performance under low signal-to-noise ratio (SNR) values [29]. There has been abundant research on these challenges, such as [30,31].

(2) Feature detection: Feature detection derives from the specific features associated with the information transmitted by a primary user. In many cases, signals have periodic statistic features such as modulation rate and carrier frequency which are usually viewed as cyclostationary characteristics. In detection, the cyclostationary characteristic of a primary user' signal can be distinguished from noise in its statistical properties such as its mean and autocorrelation [32,33]. Compared with energy detection, cyclostationary detection is not sensitive to noise uncertainty, so it has better robustness in low SNR regimes. However, this method requires more prior information on the primary user signals to decide the occupancy of primary users, so feature detection has much greater complexity [34,35].

(3) Matched filter and waveform-based detection: Matched filter detection is an optimized detection method based on the primary user's signal as prior knowledge for the secondary users. The advantage of the matched filter detection is the short time to achieve a certain probability of a false alarm or probability of a misdetection. The matched filter has a requirement of fewer signal samples, which grows as $O(1 / \mathrm{SNR})$ for a target probability of false alarm at low SNRs [36,37]. Thus, there exists a SNR wall for a matched filtering method [38]. Moreover, matched filter detection requires that the received signals are demodulated. Perfect knowledge of the primary users signal is required, so the implementation complexity of a sensing unit is impractically high [39]. To solve the SNR wall problem, waveform-based detection is proposed to improve match filtering detection when the number of samples is large enough [40]. However, information on the patterns of the primary user's signal is a prerequisite for implementing waveform-based detection, so minimizing the implementation complexity is still an open issue.

(ii) Cooperative sensing. The performance of spectrum sensing deteriorates because of noise uncertainty, shadowing, multi-path fading and the SNR wall, especially in the problem of the hidden primary user. Cooperative sensing is proposed as an efficient solution to solve the issues above, which can considerably decrease the probabilities of misdetection and false alarm, as well as improve the spectrum efficiency [41]. Furthermore, cooperative sensing can also decrease the spectrum sensing time [42]. But the challenges of cooperative sensing mainly include: the network architecture of centralized or distributed cooperation sensing [43]; detection fusion including decision fusion or data fusion [44,45]; and cooperative node selection. Furthermore, researches in $[46,47]$ have improved the cooperation sensing performance from the space diversity and the abnormality detection perspectives.

\subsection{Network information collection}

Besides the information on available spectrum, the CRN needs to collect the necessary network information such as operation type, cell coverage, and modulation type. It should also consider how to store the information on spectrum and network operation, and how to exchange the related information to meet the users' requirements.

(i) Cognitive database for network information storing and management. FCC proposed a new device for a geo-location database to assist secondary users' queries and download a coverage map of information for operation on the TV white space spectrum, which includes the spectrum bands, exact types of licensed primary services present and their specific protection requirements. A set of rules is defined in the geo-location database in $[48,49]$ to determine whether a TV channel is available for secondary users at a particular geo-graphical location, and a maximum allowed power level (per channel) at the operating location is given to avoid causing interference to primary users.

Recently, a multi-domain cognition database was pro- 
posed [50,51]. The network information in the CRN can be classified and organized to improve information management, representation and access. There are heterogeneous networks deployed in the CRN. The information from the heterogeneous networks is comprehensively related to the network type, operation frequency, location, time slot, user information, and different protocol layers of the networks. They can be classified into several domains according to their nature, and the multi-domain cognition database can store and manage them according to the domain divisions, such as the wireless domain, network domain, user domain and policy domain.

(1) The wireless domain: The wireless domain is composed of the parameters of radio transmission characteristics such as transmit power, spectrum band, Signal to Interference plus Noise Ratio (SINR), transmission rate and the radio resources bandwidth.

(2) The network domain: The network domain consists of the information reflecting the network status such as traffic, system load, network revenue, network delay, routing, scheduling scheme and node topology.

(3) The user domain: This domain focuses on information of concern to users, such as location information, quality of service (QoS) preference, user identities (IDs) and accounting.

(4) The policy domain: Policy is the guideline that manages radio resources such as communication rules and spec- trum policy.

The logical framework of a multi-domain cognition database is shown in Figure 6, which includes a local database and a cooperative database. The local database can store and manage multi-domain information from the CRN in certain regions. Local databases deployed in various regions can provide multi-domain information interaction via cooperation. The learning ability in the CRN can interpret the multi-domain information into knowledge and cases so that an intelligent prediction can be smarter and more credible.

(ii) Cognitive pilot channel for network information exchange in a CRN. As analyzed above, the cognitive ability in a CRN requires the acquisition of network information. The spectrum sensing and cognition database provides a way to collect and store the spectrum and network information. However, there is still a great challenge for cognitive ability on how to design the signaling channel to transmit network information accurately and efficiently among heterogeneous networks in a CRN. The concept of a cognitive pilot channel (CPC), proposed in the E2R and E3 projects [52], is considered one of the candidate signaling channel technologies in CRNs. The challenges facing CPC technology are summarized according to two main aspects: one is the architecture of the CPC deployment including operation mode (in-band mode, or out-band mode), information delivery mode (broadcast mode, or on-demand mode) and information structuring scheme (single layer, or

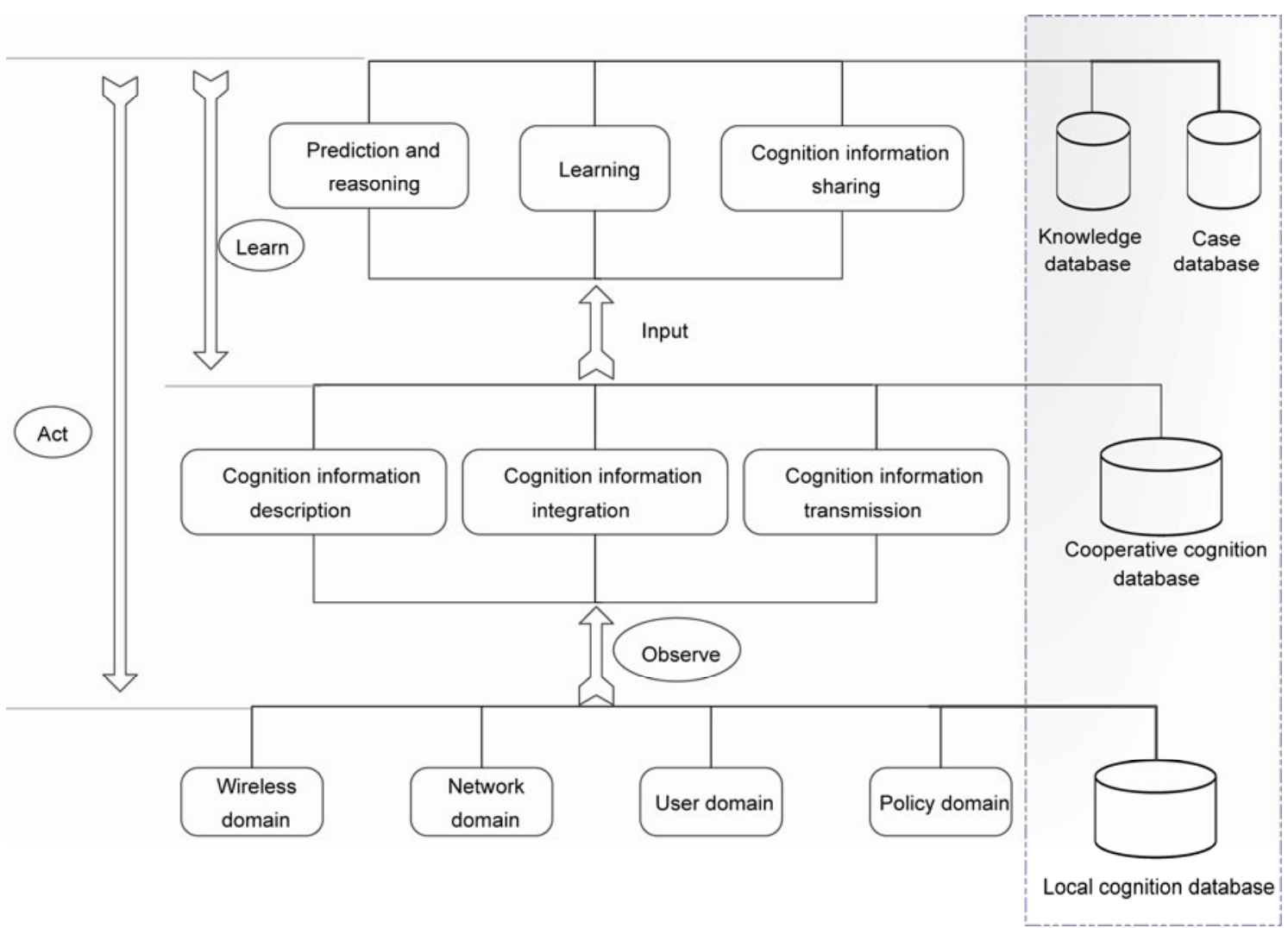

Figure 6 The logic framework of a multi-domain database in a CRN. 
multiple layers). Other aspects for the efficient cognitive information delivery process and signaling frame design are proposed in [53-55].

\section{Autonomous decision for optimized end-to- end performance}

In a CRN, an autonomous decision makes the optimized decision to overcome the challenges in wireless networks such as interference, path loss, wireless link error and link layer delay [4], and the optimized decision is considered for the network reconfiguration based on the information gathered to improve the end-to-end performance. Therefore, the autonomous decision mainly includes two important aspects as shown in Figure 7: the end-to-end performance and the optimized decision cooperating with the reconfiguration process.

\subsection{End-to-end performance}

Unlike the quality of service (QoS) in a traditional wireless network, the end-to-end performance integrates the factors from both the users and the network including the personalized requirements on wireless applications, radio resource in specific scenarios, and all network elements involved in a data transmission flow. The end-to-end performance gives a CRN its network scope, and separates it from other adaptation approaches that have only a local, single element scope [4]. Thus, the end-to-end performance will be the indicator for evaluating the performance of a CRN.

The challenge for the end-to-end performance is how to model the integrated performance requirement for the QoS of respective network elements involved in the specific end-to-end link [56]. Thus, the end-to-end performance must first map the specific performance metrics into the integrated framework as shown in Figure 8.

The QoS for the specific network elements needs to be measured and managed. All nodes in an end-to-end path have their own performance indicators. They can be classified into certain domains according to their characteristics, such as administration domain, access technology, and terminal and application domain. Furthermore, these domains have their QoS performance measurement managed by their respective servers. Servers in all domains negotiate with the end-to-end QoS server. The QoS of all the domains in the specific end-to-end link are mapped and integrated based on the game and tradeoff between the specific QoS of end-toend link. A fuzzy negotiation can then be performed to unify their description so that the performance of the different elements can be quantified and compared in a uniform metric, and the overall end-to-end performance for the specific link can be produced. Last, the integrated management policy can indicate the performance for the optimized decision or reconfiguration of the CRN.

\subsection{Optimized decision in a CRN}

Under the end-to-end performance indicator, the optimized decision can be produced based on the information detected according to the requirements of the specific scenarios. Because of the distinct requirements and cognitive ability in specific scenarios, the design for optimized decision models, including the decision model and procedure, is one of the most important challenges in CRNs. Based on the model of mathematical optimization and the different related radio resources, the optimized decision in a CRN can be classified as: spectrum decision, MAC strategies, power control,

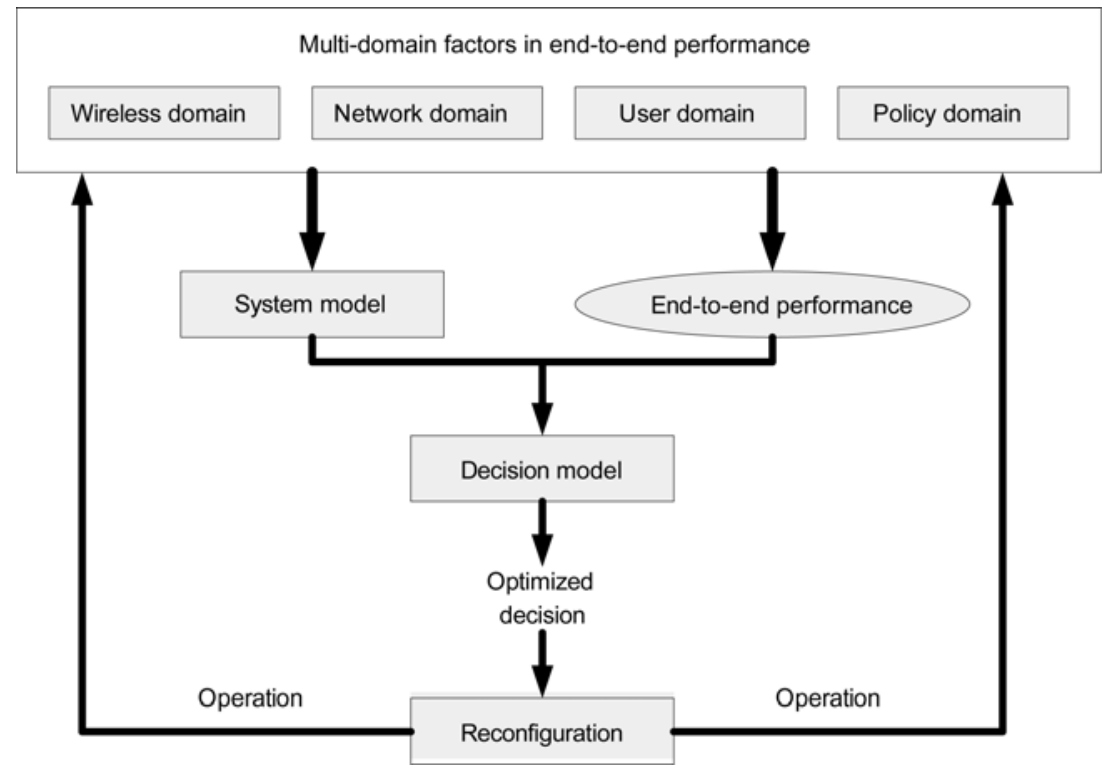

Figure 7 The autonomous decision for optimized decision making in a CRN. 


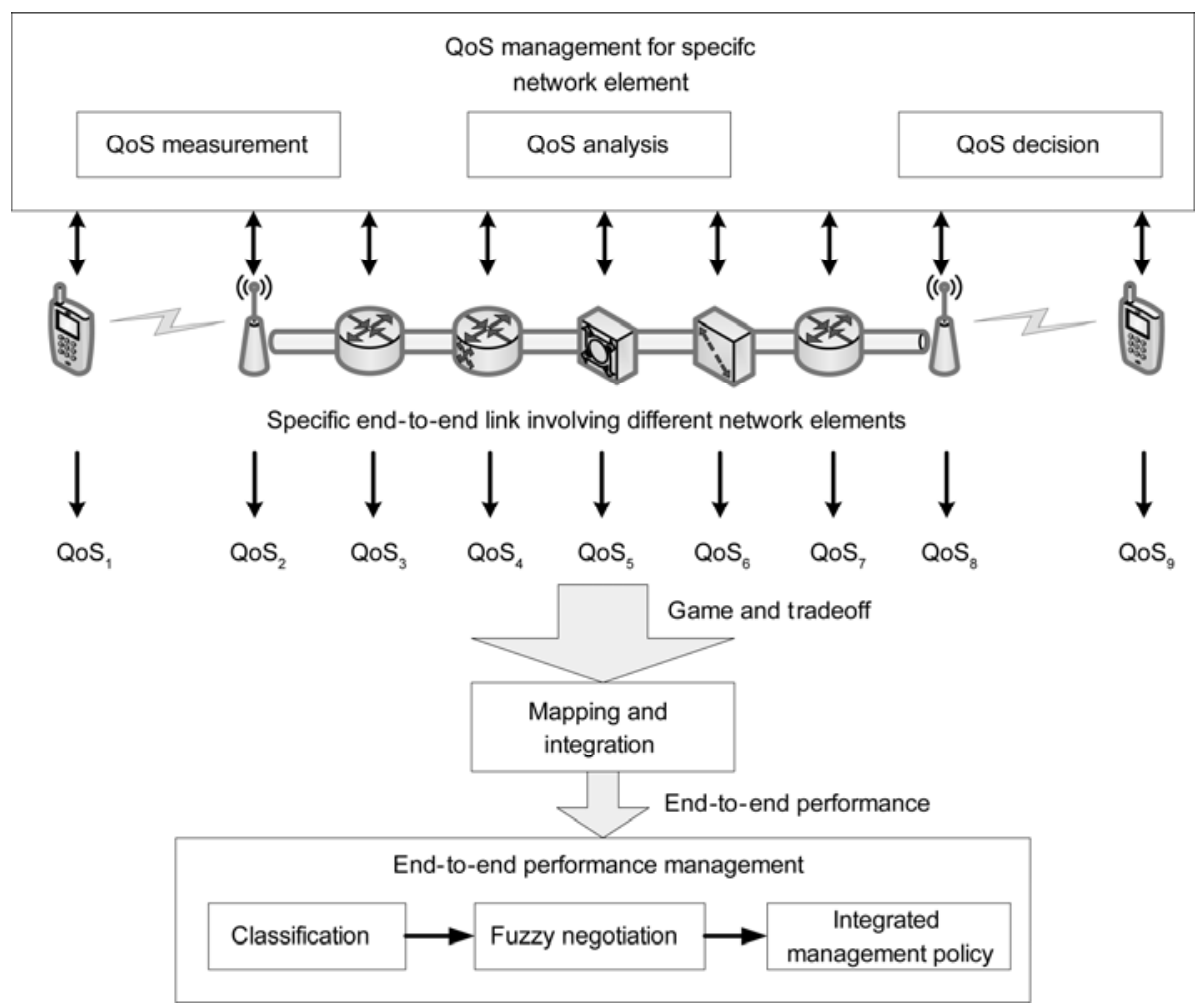

Figure 8 (Color online) The framework of end-to-end performance management.

routing in the $\mathrm{CRN}$ and relay management. Mathematical theories, such as game theory, fuzzy logic, convex optimization, and learning algorithms, are considered in the design of the decision model and procedure for these classifications.

(i) Spectrum decision. The availability and quality of the specific spectrum may change rapidly with time due to the activity of primary users or competition from other secondary users [14]. It is crucial for spectrum decisions to determine how to access the spectrum efficiently, and how to co-exist with primary users and other secondary users. The challenges for spectrum decision are mainly regarded in terms of a decision model, the architecture of spectrum sharing, the access technologies of secondary users, and spectrum mobility management.

(1) Spectrum decision model: There are mainly two categories of models for spectrum access: open spectrum sharing and hierarchical access models, as shown in Figure 9 [19]. In open spectrum sharing, the spectrum is unlicensed for both the primary and secondary users so that all users have the same right of spectrum access. Thus, the challenge is to avoid mutual interference between users. In hierarchical access, secondary users dynamically access the spectrum licensed to primary users under the constraint of causing no interference to the primary users.

Based on the above models, different mathematical theories are used to make the spectrum decision models. The Markov decision process is an efficient model for optimizing spectrum efficiency based on spectrum sensing and the prediction of the activities of primary users [57,58]. Game models have been developed to model the behavior of secondary users accessing the idle spectrum, and obtain a multiple access solution or equilibrium in the CRN [59-61]. In particular, a spectrum auction is a typical economic model for dynamic spectrum sharing [62]. Another strong model is machine learning, which decides on the available spectrum by learning from the spectrum decision experience [63].

(2) Architecture of a spectrum decision: The architectures of a spectrum decision can be classified into centralized and distributed architectures depending on whether there is a centralized network entity for spectrum decision making. The centralized entity can perform a decision algorithm to allocate the spectrum to secondary users by considering the improvement of the overall network performance $[64,65]$. The challenge for the centralized entity is to consider the tradeoff between accuracy and complexity while predicting the network load and traffic requirements $[66,67]$. Interference avoidance between heterogeneous networks should be taken into account during the decision making process $[68,69]$. In the distributed architecture, the spectrum decision is performed by each secondary user based on locally available information with no centralized control entity. Distributed secondary users must negotiate about their respective performance requirements to trade-off their selfish goal and overall system performance [70]. Thus, game theory and learning algorithms are the most popular models in designing distributed architecture for spectrum decision making [71,72]. The overhead of signaling information exchange for negotiation between secondary users 


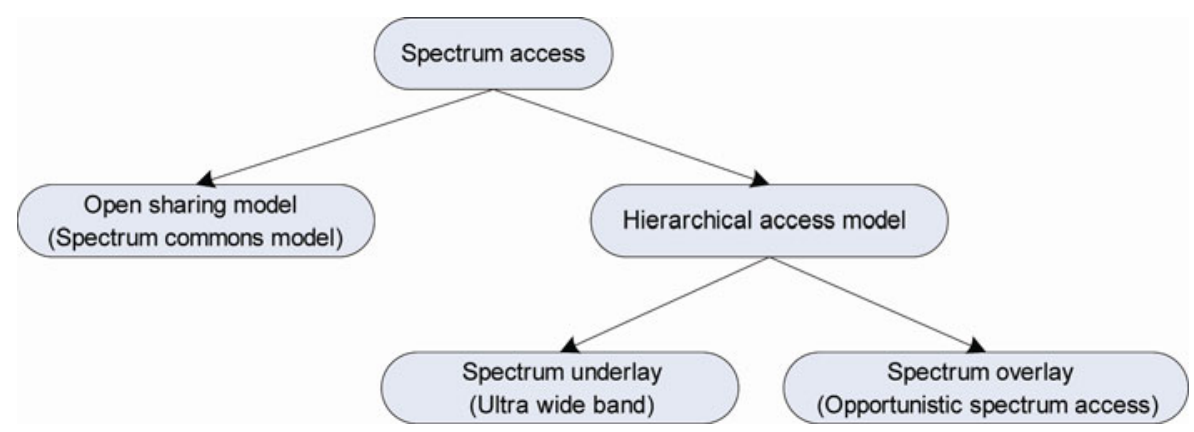

Figure 9 Spectrum access models [19].

is another important issue in distributed architecture [73].

(3) Access technology of secondary users: In the hierarchical access model, the access techniques of secondary users can be classified as spectrum underlay and overlay. In spectrum underlay, secondary users are permitted to share the licensed spectrum with primary users under the constraint that the power transmitted by secondary users will be limited under a certain threshold to avoid harmful interference to primary users [74-76]. For spectrum overlay, secondary users can only access the idle spectrum that primary users are not occupied. This is also referred to as the opportunistic spectrum access. The challenge is how to avoid access collision between secondary users and primary users due to false spectrum decision making [77,78].

(4) Spectrum mobility management: Spectrum mobility management is required to ensure the smooth and fast handoff in the changing available spectrum with minimum performance degradation while primary users retrieve the spectrum. There are crucial challenges for spectrum mobility on how to provide secondary users with smooth frequency shift and the least latency of handoff [79]. There are two categories of strategy on this issue: one is to reserve the available spectrum list which is pre-determined; the other is combined with real-time spectrum sensing, which is sensitive to the spectrum sensing ability. Both require an optimized link maintenance probability to guarantee the QoS for the secondary users [80].

(ii) MAC strategies in CRN. Medium access control (MAC) plays an important role in the functions of spectrum mobility, channel sensing, resource allocation, and spectrum sharing in CRNs [14], which refers to the policy that decides how secondary users should access a detected idle licensed spectrum band based on various network criteria. In particular, the interference between primary and secondary users must be avoided based on the new feature of the DSA in a CRN. Thus, new MAC protocols need to be designed to address new challenges in the CRN, including complexity, protocol architecture, level of cooperation within the network, control channel management and multi-channel scheduling [81-83]. Design of the reconfiguration in a CRN needs to consider the implementation of hardware-constraint MAC protocols [84].

(iii) Power control. Power control is a traditional issue of radio resource management (RRM). A water-filling algorithm is often adopted for power control. To manage the interference between secondary users and avoid the harmful interference to primary users in a CRN, a power control strategy is often jointly considered with spectrum sharing for capacity improvement in the CRN [85-87].

(iv) Routing in CRN. A changeable available spectrum imposes even greater challenges on routing schemes in a $\mathrm{CRN}$, which can be well solved by the joint consideration of the spectrum allocation. Various algorithms on the joint optimization of routing and spectrum allocation have been designed in $[88,89]$. There are also key challenges on the routing issues, including the spectrum mobility constraint, prediction on when the link may break, the joint mobility model and a topology management scheme for high mobility based on the node capacity [90,91].

(v) Cognitive relay management. Cooperative relaying is proposed to offer a more significant performance gain in these scenarios, such as coverage extension, capacity enhancement, spatial and multi-user diversity. Combined with cognitive ability, a cognitive relay can forward information in an idle spectrum that has been detected in the CRN. Recent research in this field can be classified in two categories: one is the research on spectrum sharing with primary users in a CRN where the secondary users act as the relay for primary users so that the primary users can share the licensed spectrum with them. This has been considered as a reasonable idea for realizing spectrum sharing and the details can be seen in [92,93]. The other research focuses on the use of the relay to improve CRN performance such as capacity enhancement, enlarging coverage, and reducing interference [94]. In these two research areas, key challenges such as spectrum allocation for cognitive relaying, relay-selection, and multi-hop routing have been widely investigated in [95-97].

\section{Development in the industry of CRN based on testbeds and standards}

\subsection{Testbed development for CRN}

For verification of theoretical research and a feasible proto- 
type, the development of CRN testbeds is another important issue. There are some basic requirements for CRN testbeds, including the capability of supporting multiple radios, front-ends with wide dynamic radio frequency $(\mathrm{RF})$ range to support different operation frequencies, the capability for PHY/MAC layer adaptation, fast and accurate spectrum sensing, and the capability of performing rapid reconfiguration.

Research at the University of California at Berkeley, Virginia Tech and Rutgers University, has respectively developed CRN testbeds focusing on different issues in the CRN such as the cognitive engine, fast spectrum sensing, or cross layer management which eases the design of reconfiguration and performance optimization of a cognitive protocol stack [98-101]. However, these existing CRN testbeds neither consider the networking implementation scenario in heterogeneous networks nor apply key CR technologies in a typical cellular network.

Therefore, based on the software-defined radio (SDR) platform, we design and develop the CRN testbed in Time Division Long Term Evolution (TD-LTE) cellular system, which can efficiently use the scattered vacant spectrum holes without predefined paired spectrum with sufficient frequency separation as required in LTE Frequency Division Duplex (LTE FDD) system. In order to prove the performance of key CR functions, the testbed is designed to work in two spectrum bands, including the $2.3-2.4 \mathrm{GHz}$ which is one of the candidate bands for TD-LTE system and the $698-806 \mathrm{MHz}$ band assigned to TV broadcasting system.
As shown in Figure 10, the testbed mainly consists of four parts. First, the primary system signal generator can use the software controlled signal generator equipment to generate and transmit both the radar and TV signals. Second, two cognitive eNBs (CeNBs) and eight CR users are used to verify the cellular networking and video service performance in TD-LTE system using vacant spectrum band. Third, the geo-location database will store multi-domain environment information in CRNs by updating data from local spectrum sensing results in CeNBs. Fourth, the ASM server is designed which is responsible for vacant spectrum reallocation and coordination among CeNBs in both long term and short term. On the one hand, considering the radar system which appears randomly in time-space-frequency domain with much greater transmit power on a wide spectrum band in 2.3-2.4 GHz in China, the energy detection method has been applied in the testbed to achieve a good performance with the detection probability of above $99.9 \%$ with only $1 \%$ false alarm for radar signal of $-113 \mathrm{dBm}$. Moreover, considering the network construction cost and radio signal propagation feature in much lower frequency for TD-LTE system deployment, the proposed testbed has also chosen the TV broadcasting system band around 700 $\mathrm{MHz}$ (698-806 MHz) which uses both the local spectrum sensing method in the CeNB and the geo-location database technique. Since the features of signal modulation and bandwidth for both analog and digital TV signals are known in advance and the duration of TV signals is static with a periodical pattern, the feature detection method has been

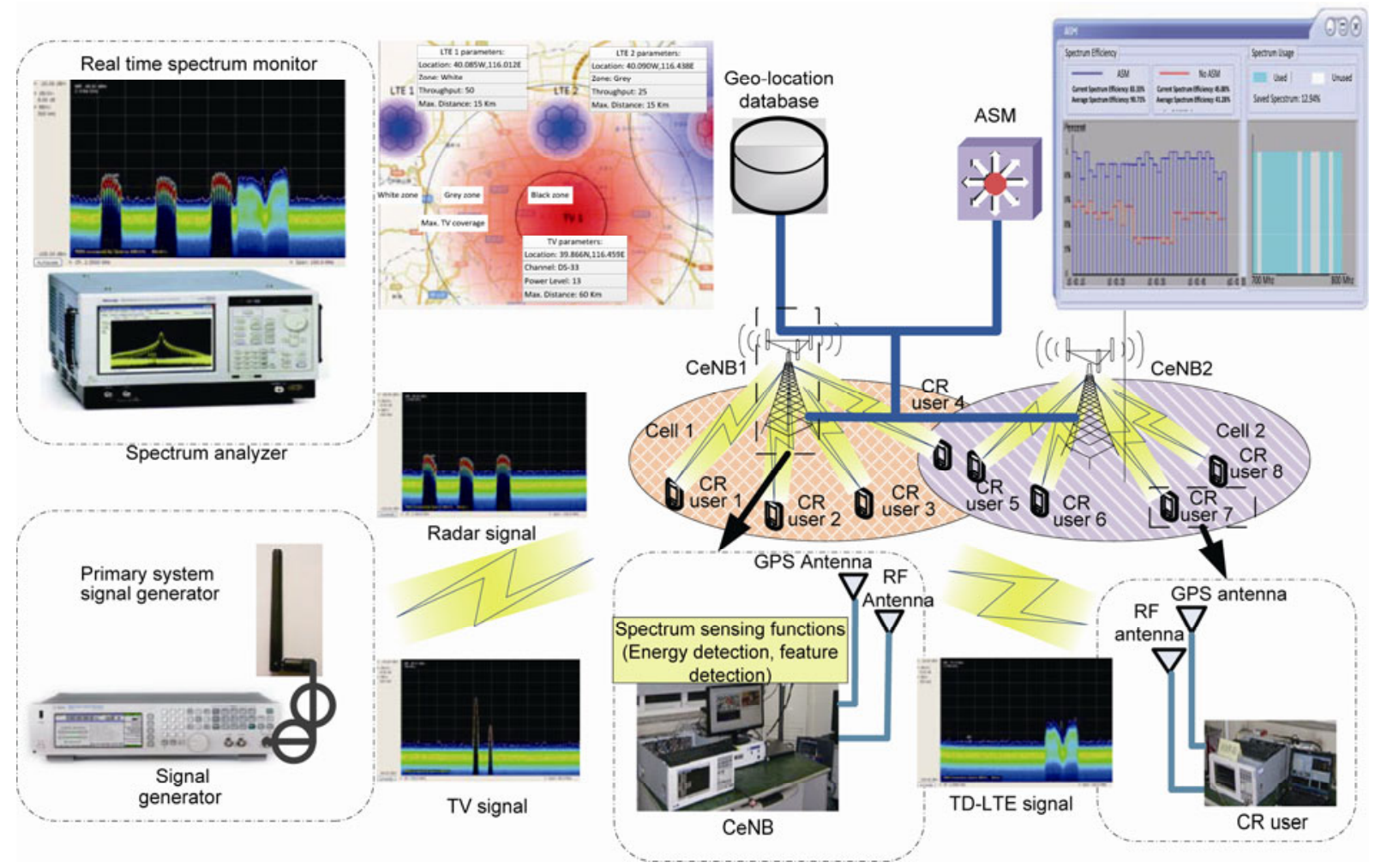

Figure 10 (Color online) TD-LTE based CRN testbed. 
used in the testbed to achieve the detection probability of above $99.9 \%$ with only $1 \%$ false alarm for TV signal of $-120 \mathrm{dBm}$, compared to $-114 \mathrm{dBm}$ by FCC. When the primary system appears (such as radar and TV signals) which can be detected by the CeNB within $1 \mathrm{~ms}$, CeNB will carry out the spectrum handover process with $\mathrm{CR}$ users within 50 ms compared to $2 \mathrm{~s}$ proposed by FCC, by using a cognitive control channel with protocol modifications based on the 3GPP Release 9 standard. In addition, the spectrum efficiency of CRNs has been improved by about $30 \%$ by using the dynamic spectrum sharing among CeNBs which are controlled by ASM server under the laboratory scenario. In summary, the proposed CRN testbed based on the TD-LTE system verifies the effectiveness of key CR functions and spectrum efficiency enhancement, which will pave the way for TD-LTE system implementation in accordance with the smooth industry develop trend of CRNs.

\subsection{Standards activities for a CRN}

The standards activities of the CRN have attracted growing attention from many researchers and industrial organizations.

The Institute of electrical and electronic engineers (IEEE) 802.22 [102] can be regarded as the first standard draft based on cognitive radio, which proposed the reuse of the idle TV spectrum without harmful interference to TV incumbents [103].

The radio group of the international telecommunications union (ITU-R) has also organized the standards activities on CRN. The ITU-R 5A is drafting a report on cognitive radio systems (CRS) in the land mobile service, and ITU-R 5D is considering how to apply the cognitive functions specific to International Mobile Telecommunications (IMT) systems [104,105].

The IEEE P1900, established under the sponsorship of IEEE SCC 41 (standards coordinating committee) in 2005, is a new standards group focusing on supporting the next generation radio and spectrum management technologies. An important focus of the standard, the P1900.4 is to provide the architecture for reconfigurable networks in a heterogeneous environment. Furthermore, the P1900.4.1 is responsible for the "Interfaces and Protocols Enabling Distributed Decision Making for Optimized Radio Resource Usage in Heterogeneous Wireless Networks". The P1900.4a is working on the "Architecture and Interfaces for Dynamic Spectrum Access Networks in White Space Frequency Bands [106,107]." Furthermore, the P1900.7 Working Group is organizing the radio interface for white space dynamic spectrum access radio systems supporting fixed and mobile operation. The detection in CRN and database technologies are also discussed in this group. Moreover, as the basis of implementing CRN, the software defined radio (SDR) forum has been leading active discussion which aims at defining standards for SDR and redirecting present regu- latory standards. This forum is involved in ensuring the accelerated proliferation of SDR technologies in wireless networks so that implementation of CRNs can make progress. Moreover, the next generation mobile networks (NGMN) alliance, which comprises most of the mobile network operators, vendors and manufacturers, launched the Innovation Day in 2011 to discuss interesting ideas on the topic of "Cognitive Radio and Small Cells".

\section{Conclusions}

Cognitive radio network technology, derived from the cognitive radio originally proposed by Mitola, has developed significantly in the last decade. The CRN extends the cognitive function into a network scope so that the challenges from the co-existence of heterogeneous wireless networks can be captured. It is a revolutionary solution contributing to the more efficient use of scarce spectrum resources and a more intelligent reconfiguration method of efficiently adapting to the changing wireless environment. Based on CRN technology, future wireless devices with more bandwidth, reliable broadband communications, and versatility for rapidly growing data applications can be realized.

In this review, the fundamental concepts and important topics on CRNs, including cognitive functions, network architecture and industry activities, are presented. In particular, recent research on the cognition cycle, cognitive ability, and spectrum decision making are reviewed in detail. From this review, readers can obtain an overview of the latest information on the framework of CRNs and its important functions. The achievements and important topics for CRNs in the future have been outlined to enable researchers to further advance the intelligence and efficiency of CRNs.

This work was supported by the National Basic Research Program of China (2009CB320400), the National Natural Science Foundation of China (61121001, 60832009), the National Science and Technology Major Project of China (2012ZX03003006) and the Program for New Century Excellent Talents in University (NCET-01-0259).

1 Akhtman J, Hanzo L. Heterogeneous networking: An enabling paradigm for ubiquitous wireless communications. Proc IEEE, 2010, 98: 135-138

2 Federal Communications Commission. Spectrum policy task force. Technical Report, ET Docket No. 2002

3 Staple G, Werbach K. The end of spectrum scarcity. IEEE Spectrum, 2004, 41: 48-52

4 Ryan W T, Daniel H F, Luiz A D, et al. Cognitive networks: Adaptation and learning to achieve end-to-end performance objectives. IEEE Commun Mag, 2007, 44: 51-58

5 Mitola J. Cognitive radio: Making software radios more personal. IEEE Pers Commun, 1999, 6: 13-18

6 Mitola J. Cognitive radio-Model-based competence for software radios. The Dissation for the Master Degree. Stockholm: Kungl Tekniska Hogskolan Royal Institute of Technology, 1999

7 Demestichas P, Dimitrakopoulos G, Strassner J, et al. Introducing reconfigurability and cognitive networks concepts in the wireless 
world. IEEE Veh Technol Mag, 2006, 1: 32-39

8 Gary J M, Joseph B E, Leon S S, et al. Cognitive radios for dynamic spectrum access-an agile radio for wireless innovation. IEEE Commun Mag, 2007, 45: 113-122

9 Haykin S. Cognitive dynamic systems. Proc IEEE, 2006, 94: 1910-1911

10 Kang G S, Hyoil K, Alexander W M, et al. Cognitive radios for dynamic spectrum access: From concept to reality. IEEE Wirel Commun, 2010, 17: 64-75

11 Haykin S. Cognitive radio: Brain-empowered wireless communications. IEEE J Sel Areas Commun, 2005, 23: 201-220

12 Allen B M, Jeffrey H R, Peter A, et al. Cognitive radio and networking research at virginia tech. Proc IEEE, 2009, 97: 660-689

13 Mitola J. Software Radio Architecture. New York: Wiley, 2000

14 Akyildiz I F, Lee W Y, Vuran M C, et al. A survey on spectrum management in cognitive radio networks. IEEE Commun Mag, 2008, 46: 40-49

15 Xin C S, Cao X J. A Cognitive Radio Network Architecture without Control Channel. In: Proceedings of Global Telecommunications Conference, 2009. Hawaii, Washington DC: IEEE, 2009. 1-6

16 Tachwali Y, Basma F, Refai $\mathrm{H} \mathrm{H}$. Cognitive radio architecture for rapidly deployable heterogeneous wireless networks. IEEE Trans Consum Electron, 2010, 56: 1426-1432

17 Sutton P D, Lotze J, Lahlou H, et al. Iris: An architecture for cognitive radio networking testbeds. IEEE Commun Mag, 2010, 48: 114122

18 Hu Z, Sun L, Tian H. A framework of access network architecture for 4G systems based on cognitive radio. In: Proceedings of Wireless Communications, Networking and Mobile Computing, 2009, Beijing. 24-26

19 Zhao Q, Sadler B M. A survey of dynamic spectrum access. IEEE Signal Process Mag, 2007, 24: 79-89

20 Feng Z Y, Li W, Li Q, et al. Dynamic spectrum management for WCDMA/DVB heterogeneous systems. IEEE Trans Wirel Commun, 2011, 10: 1582-1593

21 Le V B, Feng Z Y, Bourse D, et al. A cell based dynamic spectrum management scheme with interference mitigation for cognitive networks. Wireless Pers Commun, 2009, 49: 275-293

22 Joseph M, Attar A, Zhang H H O, et al. Achievements and the road ahead: The first decade of cognitive radio. IEEE Trans Veh Technol, 2010, 59: 1574-1578

23 Wang B B, Liu K J R. Advances in cognitive radio networks: A survey. IEEE J Sel Top Sign Proces, 2011, 5: 5-24

24 Haykin S, Thomson D J, Reed J H. Spectrum sensing for cognitive radio. Proce IEEE, 2008, 97: 849-877

25 Kolodzy P. Next generation communications: Kickoff meeting. In: Proceedings of DARPA, 2001

26 Yucek T, Arslan H. A survey of spectrum sensing algorithms for cognitive radio applications. IEEE Commun Surv Tutor, 2009: 116-130

27 Establishement of interference temperature metric to quantify and manage interference and to expand available unlicensed operation in certain fixed mobile and satellite frequency bands, FCC, 2003, FCC Doc. ET Docket: 03-289

28 Yucek T, Arslan H. Spectrum characterization for opportunistic cognitive radio systems. In: Proceedings of Military Communication Conference, 2006, Washington DC: IEEE, 2006. 1-6

29 Quan Z, Shellhammer S J, Zhang W, et al. Spectrum sensing by cognitive radios at very low SNR. In: Proceedings of Global Communications Conference, 2009, Beijing, Washington DC: IEEE, 2009. 1-6

30 Tang H. Some physical layer issues of wide-band cognitive radio systems. In: Proceedings of International Symposium on New Frontiers in Dynamic Spectrum Access Networks, 2005, Baltimore, Maryland, Washington DC: IEEE, 2005. 151-159

31 Weidling F, Datla D, Petty V, et al. A framework for RF spectrum measurements and analysis. In: Proceedings of International Symposium on New Frontiers in Dynamic Spectrum Access Networks, 2005, Baltimore, Maryland, Washington DC: IEEE, 2005. 573-576

32 Lehtomaki L, Vartiainen J, Juntti M, et al. Spectrum sensing with forward methods. In: Proceedings of Military Communications Conference, 2006, Washington DC: IEEE, 2006. 1-7

33 Gardner U W. Exploitation of spectral redundancy in cyclostationary signals. IEEE Signal Process Mag, 1991, 8: 14-36

34 Muraoka K, Ariyoshi M, Fujii T. A novel spectrum-sensing method based on maximum cyclic autocorrelation selection for cognitive radio system. In: Proceedings of 3rd Symposium on New Frontiers in Dynamic Spectrum Access Networks, 2008, Dublin, Washington DC: IEEE, 2008. 1-7

35 Du K L, Wai H M. Affordable cyclo-stationarity-based spectrum sensing for cognitive radio with smart antennas. IEEE Trans Veh Technol, 2010, 59: 1877-1887

36 Proakis J G. Digital Communications. 4th ed. New York: McGrawHill, 2001

37 Tandra R, Sahai A. Fundamental limits on detection in low SNR under noise uncertainty. In: Proceedings of International Conference on Wireless Networks, Communication and Mobile Computing, 2005, Maui, HI, Washington DC: IEEE, 2005. 464-469

38 Tandra R, Sahai A. SNR walls for signal detection. IEEE J Sel Top Sign Proces, 2008, 2: 4-17

39 Cabric D, Mishra S, Brodersen R. Implementation issues in spectrum sensing for cognitive radios. In: Proceedings of Asilomar Conference on Signals, System, Computation, 2004, Washington DC: IEEE, 2004. 772-776

40 Cabric D, Tkachenko A, Brodersen R. Spectrum sensing measurements of pilot, energy, and collaborative detection. In: Proceedings of Military Communications Conference, 2006, Washington DC: IEEE, 2006. 1-7

41 Wang Z L, Feng Z Y, Zhang P. An iterative hungarian algorithm based coordinated spectrum sensing strategy. IEEE Commun Lett, 2011, 15: 49-51

$42 \mathrm{Hu}$ W, Willkomm D, Abusubaih M, et al. Dynamic frequency hopping communities for efficient IEEE 802.22 operation. IEEE Commun Mag, 2007, 45: 80-87

43 Hillenbrand J, Weiss T, Jondral F. Calculation of detection and false alarm probabilities in spectrum pooling systems. IEEE Commun Lett, 2005, 9: 349-351

44 Liang Y C, Zeng Y H, Peh E, et al. Sensing-throughput tradeoff for cognitive radio networks. IEEE Trans Wireless Commun, 2008, 7: 1326-1337

45 Meng J, Yin W T, Li H S, et al. Collaborative spectrum sensing from sparse observation in cognitive radio networks. IEEE J Sel Areas Commun, 2011, 29: 327-337

46 Duan D L, Yang L Q, Principe J C. Cooperative diversity of spectrum sensing for cognitive radio systems. IEEE Trans Signal Process, 2010, 58: 3218-3227

$47 \mathrm{Li} \mathrm{H} \mathrm{S,} \mathrm{Zhu} \mathrm{H.} \mathrm{Catch} \mathrm{me} \mathrm{if} \mathrm{you} \mathrm{can:} \mathrm{An} \mathrm{abnormality} \mathrm{detection} \mathrm{ap-}$ proach for collaborative spectrum sensing in cognitive radio networks. IEEE Trans Wireless Commun, 2010, 9: 3554-3565

48 Federal Communications Commission. Memorandum Opinion and Order on Reconstruction of the Seventh Report and Order and Eighth Report. 2008, 3, Rep. 08-72

49 Gurney D, Buchwald G, Ecklund L, et al. Geo-location database techniques for incumbent protection in the TV white space. In: Proceedings of 3rd Symposium on New Frontiers in Dynamic Spectrum Access Networks, 2008, Chicago, Washington DC: IEEE, 2008. 1-9

50 ITU-R Document 5A/377-E. Cognitive radio systems in the land mobile service multi-dimension cognitive database for cognitive radio system. 2009, 11

$51 \mathrm{Xu}$ Y, Feng Z Y, Zhang P. Research on Cognitive Wireless Networks: Theory, Key Technologies and Testbed. In: Proceedings of 6th International ICST Conference on Cognitive Radio Oriented Wireless Networks and Communications, 2011, Osaka, Washington DC: IEEE, 2011. 291-295

52 Cordier P, Houze P, Jema S B, et al. E2R Cognitive Pilot Channel concept. In: Proceedings of 15th IST Mobile and Wireless Communications Summit, 2006, Mykonos Island, 2006. 1-4

53 Sallent O, Perez R J, Agusti R, et al. Cognitive pilot channel enabling spectrum awareness. In: Proceedings of IEEE International Confer- 
ence on Communications Workshops, 2009, Dresden, Washington DC: IEEE, 2009. 1-6

54 Zhang Q X, Feng Z Y, Zhang G Y, et al. Efficient mesh division and differential information coding schemes in broadcast cognitive pilot channel. Wireless Pers Commun, 2012, 63: 363-392

55 Feng Z Y, Zhang Q X, Tian F, et al. Novel research on cognitive pilot channel in cognitive wireless network. Wireless Pers Commun, 2012, 62: $455-478$

56 Dong X Y, Wang J J, Zhang Y, et al. End-to-end QoS provisioning in future cognitive heterogeneous networks. In: Proceedings of IEEE International Conference on Communications Technology and Applications, 2009, Beijing, Washington DC: IEEE, 2009. 425-429

57 Zhao Q, Tong L, Swami A, et al. Decentralized cognitive MAC for opportunistic spectrum access in ad hoc networks: A POMDP framework. IEEE J Sel Areas Commun, 2007, 25: 589-600

58 Chen Y, Zhao Y, Swami A. Distributed spectrum sensing and access in cognitive radio networks with energy constraint. IEEE Trans Signal Process, 2009, 57: 783-797

59 Srivastava V, Neel J A, MacKenzie A B, et al. Using game theory to analyze wireless ad hoc networks. IEEE Commun Surv Tutor, 2005, 7: 46-56

60 Zhang T, Yu X Y. Spectrum sharing in cognitive radio using game theory-A survey. In: Proceedings of 2010 6th International Conference on Wireless Communications Networking and Mobile Computing, Chengdu, 2010. 1-5

$61 \mathrm{Li} \mathrm{J}$, Chen D, Li W, et al. Multiuser power and channel allocation algorithm in cognitive radio. In: Proceedings of International Conference on Parallel Processing, Xi' an. 2007. 1-6

62 Huang J W, Berry R A, Honig M L. Auction-based spectrum sharing. Springer Mobile Netw Appl, 2006, 11: 405-418

63 Clancy C, Hecker J, Stuntebeck E, et al. Applications of machine learning to cognitive radio networks. IEEE Wirel Commun, 2007, 14: $47-52$

64 3rd Generation Partnership Project. Technical Specification Group Services and Systems Aspects; TS 23.251 v6.6.0, Network Sharing; Architecture and functional description (Release 6). 2006, 3

65 Kulkarni R, Zekavat S A. Traffic aware inter-vendor dynamic spectrum allocation: Performance in multi-vendor environment. In: Proceedings of International Communications Conference on Mobile Computation, Vancouver: 2006. 1-5

66 Salami G, Durowoju O, Attar A H, et al. A comparison between the centralized and distributed approaches for spectrum management. IEEE Commun Surv Tutor, 2011, 13: 274-291

67 Feng H, Shu Y. Study on network traffic prediction techniques. In: Proceedings of International Conference on Wireless Communication. Networks. Wuhan: 2005, 23-26

68 Pereirasamy M K. Dynamic inter-operator spectrum sharing for UMTS FDD with displaced cellular networks. In: Proceedings of Wireless Communications and Networking Conference, 2005, New Orleans, Washington DC: IEEE, 2005. 1720-1725

69 Le V, Lin Y, Wang X, et al. A cell based dynamic spectrum management scheme with interference mitigation for cognitive networks. In: Proceedings of Vehicular Technology Conference, 2008, Singapore, Washington DC: IEEE, 2008. 1594-1598

70 Cao L, Zheng H. Distributed spectrum allocation via local bargaining. In: Proceedings of Sensor and Ad Hoc Communications and Networks, 2005, California, Washington DC: IEEE, 2005. 475-486

71 Zhu J, Liu K L R. Dynamic spectrum sharing: A game theoretical overview. IEEE Commun Mag, 2007, 45: 88-94

72 Musku M R, Cotae P. Cognitive radio: Time domain spectrum allocation using game theory. In: Proceedings of International Conference on System of Systems Engineering, 2007, San Antonio, TX, Washington DC: IEEE, 2007. 1-6

73 Asterjadhi A, Baldo N, Zorzi M. A distributed network coded control channel for multihop cognitive radio networks. IEEE Network, 2009, 23: $26-32$

74 Long B L, Hossain E. Resource allocation for spectrum underlay in cognitive radio networks. IEEE Trans Wireless Commun, 2008, 7: 5306-5315
75 Zheng H, Peng C. Collaborative and fairness in opportunistic spectrum access. In: Proceedings of International Conference on Communications, 2005, Seoul, Washington DC: IEEE, 2005. 3132-3136

76 Xing Y, Mathur C N, Haleem M A, et al. Dynamic spectrum access with QoS and interference temperature constraints. IEEE Trans Mobile Computation, 2007, 6: 423-433

77 Liang Y C, Zeng Y, Peh E C Y, et al. Sensing-throughput tradeoff for cognitive radio networks. IEEE Trans Wireless Commun, 2008, 7: 1326-1337

78 Huang S, Liu X, Ding Z. Optimal transmission strategies for dynamic spectrum access in cognitive radio networks. IEEE Trans Mobile Computing, 2009, 8: 1636-1648

79 Zhang Y. Spectrum handoff in cognitive radio networks: Opportunistic and negotiated situations. In: Proceedings of International Conference on Communications, 2009, Dresden, Washington DC: IEEE, 2009. 1-6

80 Wang L C, Anderson C. On the performance of spectrum handoff for link maintenance in cognitive radio. In: Proceedings of International Symposium on Wireless Pervasive Computing, 2008, Santorini, Washington DC: IEEE. 670-674

81 Xiao Y, Hu F. Cognitive Radio Network. London: Taylor \& Francis Group, 2009

82 Jia C C, Zhang Q. Hardware-constrained multi-channel cognitive MAC. In: Proceedings of Global Telecommunications Conference, 2007, Washington DC: IEEE, 2007. 4653-4658

83 Ghaboosi K, Latva-aho M, Xiao Y. A distributed multi-channel cognitive MAC protocol for IEEE 802.11s wireless mesh networks. In: Proceedings of International Conference on Cognitive Radio Oriented Wireless Networks and Communications, 2008, Singapore, Washington DC: IEEE, 2008. 1-8

84 Gu S, Xu P C, Wang X B, et al. A real time testbed for the evaluation of cognitive radio MAC. In: Proceedings of 2010 Global Telecommunications Conference, 2010, Florida, Washington DC: IEEE, 2010. $1-5$

85 Chen Y, Yu G, Zhang Z, et al. On cognitive radio networks with opportunistic power control strategies in fading channels. IEEE Trans Wireless Commun, 2008, 7: 2752-2761

$86 \mathrm{Xu} \mathrm{D}$, Feng Z Y, Zhang P. Protecting primary users in cognitive radio networks with effective capacity loss constraint. IEICE Trans Commun, 2012, 95: 349-353

87 Xu D, Feng Z Y, Li Y Z, et al. Optimal power control of cognitive radio under SINR constraint with primary user's cooperation. IEICE Trans Commun, 2011, 94: 2685-2689

88 Wang Q, Zheng H. Route and spectrum selection in dynamic spectrum networks. In: Proceedings of IEEE Consumer Communication Networks Conference, 2006, Las Vegas, Washington DC: IEEE, 2006. 625-629

89 Xia B, Wahab M H, Yang Y, et al. Reinforcement learning based spectrum-aware routing in multi-hop cognitive radio networks. In: Proceedings of International Conference on Cognitive Radio Oriented Wireless Networks and Communication, 2009, Hannover, 1-5

90 Suyang J, Evans J B. Mobility-aware routing protocol for mobile Ad-Hoc networks. In: Proceedings of IEEE International Conference on Communications Workshops, 2009, Dresden, Washington DC: IEEE, 2009. 1-6

91 Bhandari V, Vaidya $\mathrm{N} \mathrm{H}$. Connectivity and capacity of multi-channel wireless networks with channel switching constraints. In: Proceedings of IEEE International Conference on Computer Communications, 2007, Anchorage, Washington DC: IEEE, 2007. 785-793

92 Pandharipande A, Keong H C. Spectrum pool reassignment for a cognitive OFDM-based relay system. In: Proceedings of International Conference on Cognitive Radio Oriented Wireless Networks and Communications, Orlando, 2008. 90-94

93 Yang H, Ting S H, Pandharipande A. Cooperative spectrum sharing with distributed secondary user selection. In: Proceedings of International Conference on Communications, 2009, Dresden, Washington DC: IEEE, 2009. 1-5

94 Zhang Q X, Feng Z Y, Zhang P. Joint cooperative relay scheme for spectrum-efficient usage and capacity improvement in cognitive ra- 
dio networks. EURASIP J Wirel Commun Netw, 2012, 37: 1-9

95 Song C Y, Shiba H, Shimamoto S. A cognitive relay approach for multi-hop wireless ad hoc communication. In: Proceedings of International Conference on Communications and Networking in China, Hangzhou, 2008. 1230-1234

96 Jia J, Zhang J, Zhang Q. Cooperative relay for cognitive radio networks. In: Proceedings of International Conference on Computer Communications, Rio de Janeiro, 2009. 2304-2312

97 Xiao Y, Bi G A, Niyato D. Game theoretic analysis for spectrum sharing with multi-hop relaying. IEEE Trans Wireless Commun, 2011, 10: 1527-1537

98 Mishra S, Cabric D, Chang C, et al. A real time cognitive radio testbed for physical and link layer experiments. In: Proceedings of International Symposium on New Frontiers in Dynamic Spectrum Access Netw, 2005, Baltimore, Washington DC: IEEE, 2005. 562-567

99 Rieser C, Rondeau T, Bostian C, et al. Cognitive radio testbed: Further details and testing of a distributed genetic algorithm based cognitive engine for programmable radios. In: Proceedings of Military Communication Conference, 2005, Atlantic, Washington DC: IEEE, 2005. 1437-1443

100 Jongmin P, Kim K W, Song T, et al. A cross-layer cognitive radio testbed for the evaluation of spectrum sensing receiver and interference analysis. In: Proceedings of International Conference on Cognitive Radio Oriented Wireless Networks and Communications, Singapore, 2008. 1-6

101 Jia J C, Zhang Q. A testbed development framework for cognitive radio networks. In: Proceedings of International Conference on Com- munications, Dresden, Washington DC: IEEE, 2009. 1-5

102 Cordeiro C, Challapali K, Birru D, et al. IEEE 802.22: The first worldwide wireless standard based on cognitive radios. In: Proceedings of International Symposium on New Frontiers in Dynamic Spectrum Access Netw, Baltimore, Washington DC: IEEE, 2005. 328-337

103 Poston J, Horne W. Discontiguous OFDM considerations for dynamic spectrum access in idle TV channels. In: Proceedings of International Symposium on New Frontiers in Dynamic Spectrum Access Netw, 2005, Baltimore, Washington DC: IEEE, 2005. 607-610

104 ITU-R 5A/383-E. Proposed modifications to the working document towards a preliminary draft new report: Cognitive Radio Systems in the Land Mobile Service. In: 4th Meeting of Working Party 5A, Geneva, 2009 Nov. 23-Dec.2

105 ITU-R 5D/647-E. Proposal on the workplan and draft working document on the CRS specitic for IMT systems: Cognitive Radio Systems Specific for IMT Systems. In: 7th Meeting of Working Party 5D, Turin, 2010 Feb. 17-24

106 Muck M, Buljore S, Martigne P, et al. IEEE P1900. B: Coexistence support for reconfigurable, heterogeneous air interfaces. In: Proceedings of International Symposium on New Frontiers in Dynamic Spectrum Access Networks, 2007, Dublin, Washington DC: IEEE, 2007. 381-389

107 Filin S, Harada $\mathrm{H}$, Murakami $\mathrm{H}$, et al. IEEE draft standards P1900.4.1 and P1900.4a for heterogeneous type and spectrum sharing type cognitive radio systems. In: Proceedings of International Symposium on Personal, Indoor and Mobile Radio Communications, Istanbul, Washington DC: IEEE, 2010. 1-6

Open Access This article is distributed under the terms of the Creative Commons Attribution License which permits any use, distribution, and reproduction in any medium, provided the original author(s) and source are credited. 